\title{
LARGE-SCALE STRUCTURE IN THE DURHAM/UKST GALAXY REDSHIFT SURVEY
}

\author{
A. BROADBENT ${ }^{1}$, T. SHANKS ${ }^{1}$, F.G. WATSON ${ }^{2}$, Q.A. PARKER ${ }^{3}$, R. FONG ${ }^{1}$, \\ A.P. OATES ${ }^{2}$ and C.A. COLLINS ${ }^{1}$ \\ ${ }^{1}$ Physics Department, University of Durham, South Road, Durham DHI 3LE, U.K. \\ ${ }^{2}$ Royal Greenwich Observatory, Madingley Road, Cambridge CB3 OHA, U.K. \\ ${ }^{3}$ Anglo-Australian Observatory, PO Box 296, Epping NSW 2121, Australia
}

\begin{abstract}
We report on the progress of the compilation and analysis of the Durham/UKST galaxy redshift survey. This survey will probe a large contiguous volume of space within a $1500 \mathrm{sq}$. deg. area of sky around the SGP. It will contain redshifts of $\sim 4000$ galaxies of $b_{3}<17^{\mathrm{m}}$ providing detailed information about the structure of the Universe on large scales. Large features on scales of $\sim 100 \mathrm{~h}^{-1} \mathrm{Mpc}$ are clearly visible on examination of the completed section of the survey, although a statistical analysis of the survey by means of the two-point correlation function is close to zero on scales of $r>10 \mathrm{~h}^{-1} \mathrm{Mpc}$.
\end{abstract}

By compiling the redshifts of a high proportion of the galaxies in a large, contiguous volume of space, the principal aim of the Durham/UKST galaxy redshift survey is to enable detailed study of structure in the universe over a wide range of scales. Accordingly, in a 1500 sq. deg. area around the SGP, galaxies are randomly sampled at a rate of 1-in-3 from the Edinburgh/Durham Southern Galaxy Catalogue (Collins et al. 1988) down to a limiting magnitude of $b_{J} \sim 17^{\mathrm{m}}$. The completed survey will contain $\sim 4000$ galaxy redshifts probing to a depth of $\sim 300 \mathrm{~h}^{-1} \mathrm{Mpc}$ in a volume of $\sim 4 \times 10^{6} h^{-1} \mathrm{Mpc}^{3}$ (where $h$ is the Hubble's Constant in units of $100 \mathrm{kms}^{-1} \mathrm{Mpc}^{-1}$ ). This should allow us to study the galaxy distribution on scales of $1 h^{-1}$ to $100 h^{-1} \mathrm{Mpc}$.

The galaxy redshifts are obtained using the fibre-linked spectroscopy system, FLAIR (see Parker \& Watson, this volume) on the UK Schmidt Telescope in Australia. The system has two plate-holders, one with 92 and the other with $73100 \mu \mathrm{m}$ fibre bundles. To obtain a high percentage $(>80 \%)$ of the redshifts of the galaxy sample on any one field we typically require $\sim 5 \times 3000$ s exposures and therefore with only a 40-60 minute turn around time in changing plate-holders, it is possible to gather $\sim 150$ galaxy redshifts in one night. The recent implementation of the two plate-holders enables a large project such as that described here to be completed in a reasonable period of time and remain competitive with other large redshift surveys under construction elsewhere. Also, FLAIR will tolerate seeing conditions which are too poor for photographic survey work, thus making efficient use of the telescope.

So far, 26 of the $605^{\circ} \times 5^{\circ}$ fields have been observed and a 1-in-3 sample will be drawn from the 1-in-1 sample of Parker (1992) which covers 9 fields at $\delta-35^{\circ}$ and has a similar magnitude limit (see Fig. 1). Twelve fields along a $60^{\circ} \times 5^{\circ}$ strip at $\delta-30^{\circ}$ have been fully reduced and we have deduced a total of 544 galaxy redshifts to an accuracy of $\pm 150 \mathrm{kms}^{-1}$.

The cone plot in Fig. 2 represents the positions of the galaxies in RA and velocity. On inspection of this plot it can be seen that there are apparently large structures in the distribution 


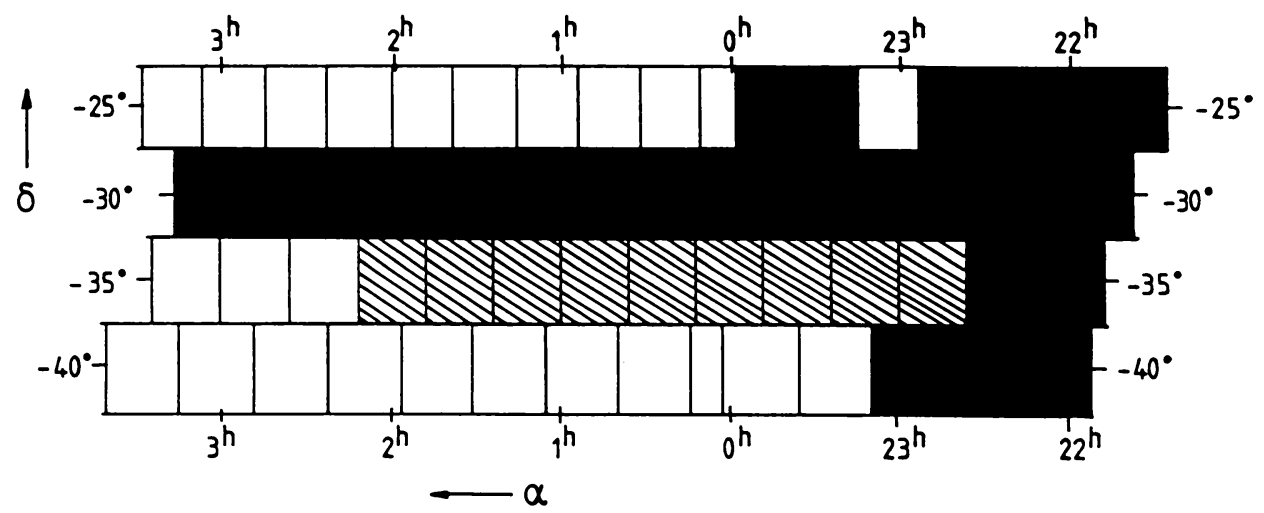

Figure 1. A plan of the Durham/UKST galaxy redshift survey area showing the individual field areas, those which have been observed (solid) and those which have been observed by Parker (hatched). The reduction and analysis described in this paper has been performed on galaxies which lie in the $\delta-30^{\circ}$ strip between $21^{\text {b }} 39$ to $02^{\text {h }} 07$.

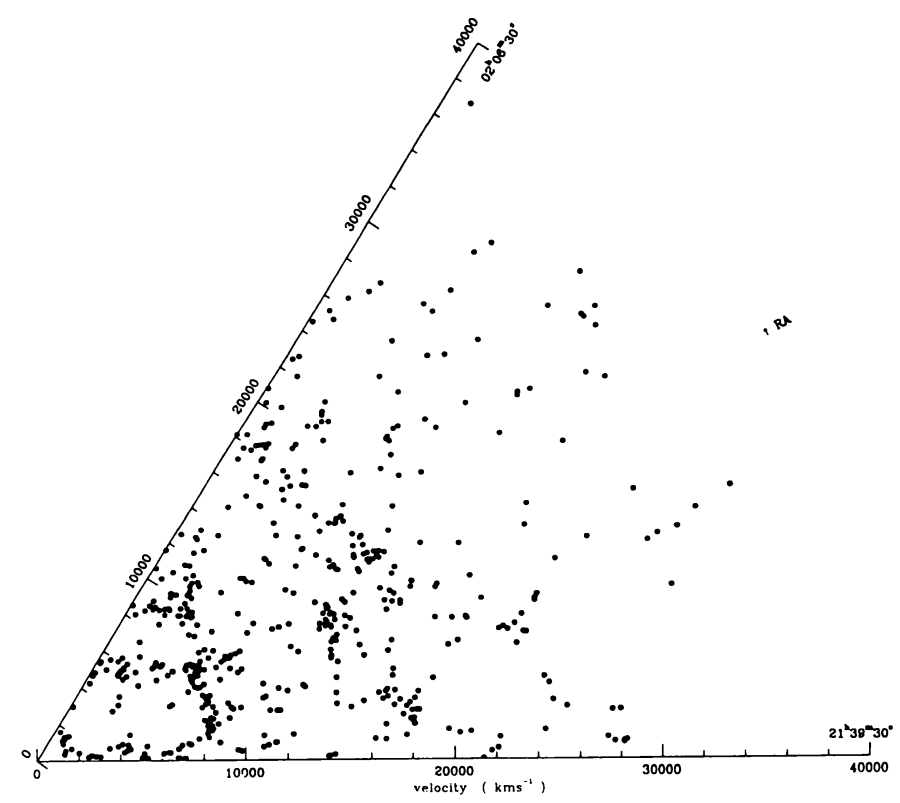

Figure 2. A cone-plot of the galaxy distribution in a $60^{\circ} \mathrm{RA}$ strip at $\delta=-30^{\circ}$ from the Durham/UKST redshift survey. Redshift increases across the page and RA increases up the page. Strong filamentary clustering on scales of $\sim 100 \mathrm{~h}^{-1} \mathrm{Mpc}$ can be seen along and across the line of sight. 


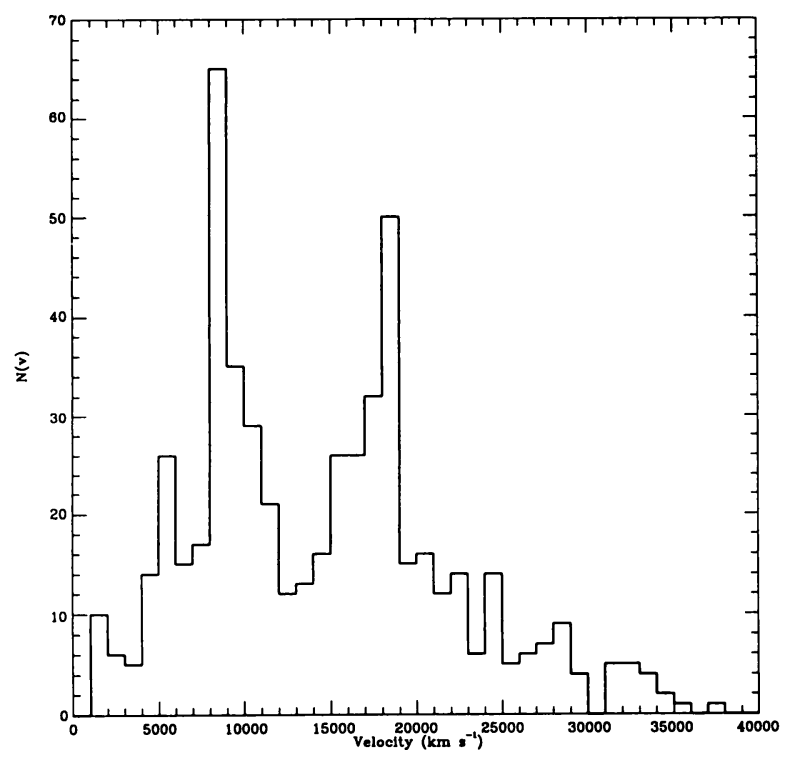

Figure 3. A redshift histogram of the galaxies in the Durham/UKST survey. There are two prominent peaks at $\sim 8000$ and $\sim 1800 \mathrm{kms}^{-1}$. Again, the galaxy distribution looks inhomogeneous on scales of $100 h^{-1}$.
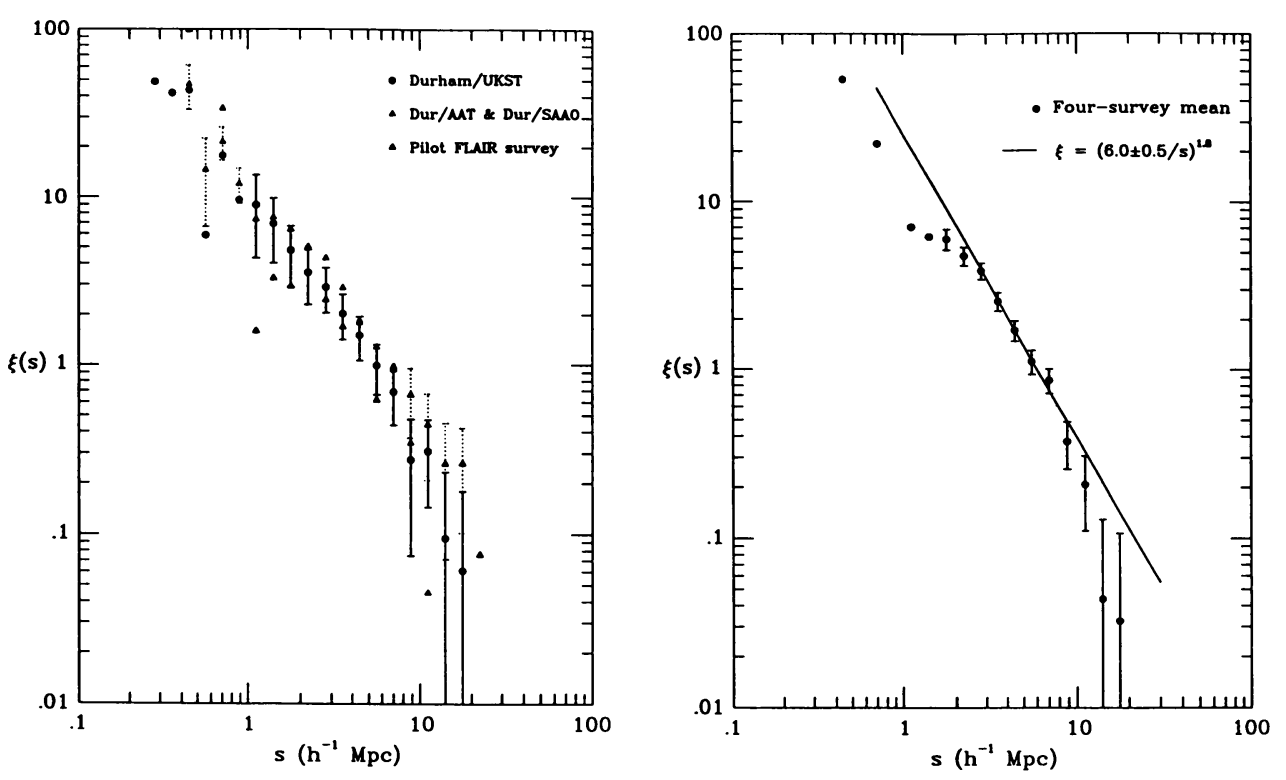

Figure 4. (a) The small scale redshift two-point correlation function from the Durham/UKST galaxy redshift survey. Errors have been estimated from a consideration of the field-to-field variations in the function. Also, for comparison the $\xi$ derived from the Durham/AAT, Durham/SAAO and pilot FLAIR surveys are shown with representative errors. (b) The average $\xi$, from all four surveys. The solid line is a 1.8 power law approximation to the form of $\xi_{x}$, between 2 and $7 h^{-1} \mathrm{Mpc}$. The error on the amplitude is estimated from the survey-to-survey variation. 

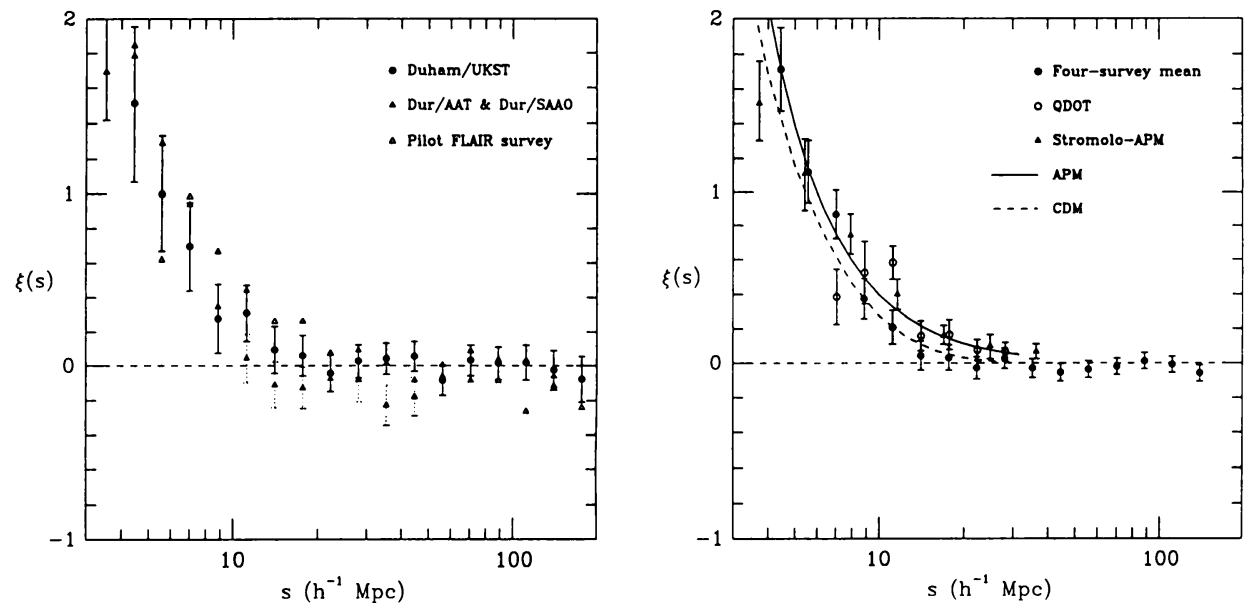

Figure 5. (a) The redshift two-point correlation function at large scales derived from galaxies in the Durham/UKST redshift survey, the Durham/AAT, Durham/SAAO and pilot FLAIR surveys. (b) The error weighted mean large scale $\xi$, of the four redshift surveys ( 1300 galaxies). The open circles represent the correlation function calculated from IRAS galaxies and the triangles that from the APM/Stromlo redshift survey. The solid line is an approximation to $\xi$ derived from the APM angular two-point correlation function, $\omega(\theta)$ and the dashed line is the predicted $\xi$ from standard CDM theory. Our results show no significant power beyond $10 h^{-1} \mathrm{Mpc}$ although we cannot rule out the APM result at more than the $2 \sigma$ level.

on scales of $\sim 100 h^{-1} \mathrm{Mpc}$. Among the most noticeable of these are the long filamentary structures surrounding a region of low density between 0 and $8000 \mathrm{~km} \mathrm{~s}^{-1}$ which has been referred to as the Sculptor void (Fairall \& Jones 1988). Structures are discernible at higher redshifts, in particular, the possible wall structure at $\sim 18000 \mathrm{kms}^{-1}$. Not surprisingly, the $\mathrm{n}(\mathrm{z})$ histogram plot in Fig. 3 has two strong peaks at 8000 and $18000 \mathrm{kms}^{-1}$.

We have made a statistical investigation of the nature of the galaxy distribution in this RA slice by calculating the redshift two-point correlation function, $\xi_{s}(s)$. This is a measure in velocity space of the excess probability of finding a galaxy in a volume element over a random and homogeneous distribution and was calculated using the methods of Shanks et al. (1983). In Figs. $4 \mathrm{a}$ and $5 \mathrm{a}$ is shown $\xi_{s}$ on small and large scales respectively and in comparison those derived from the Durham/AAT, Durham/SAAO and pilot FLAIR surveys (Peterson et al. 1986; Metcalfe et al. 1989; Watson et al. 1991). All are in reasonably good agreement within the errors.

The results of all four surveys are readily combined to produce an error weighted mean which effectively samples 1300 galaxy redshifts within a volume of $\sim 2 \times 10^{6} \mathrm{Mpc}^{3}$. The resulting mean $\xi_{c}$ is shown on large and small scales in Figs. $4 \mathrm{~b}$ and $5 \mathrm{~b}$ respectively.

The straight line in Fig. $4 \mathrm{~b}$ is a power law approximation to $\xi_{s}$, between 2 and $7 h^{-1} \mathrm{Mpc}$. The deviation from a pure power law between 1 and $2 h^{-1}$ Mpc was remarked upon in the AAT and SAAO surveys alone and an analysis of the correlation function in directions along and perpendicular to the line of sight should help to clarify whether this is due to peculiar motion and 
infall or true, non-scale-free clustering of galaxies on small scales.

On larger scales $>10 h^{-1} \mathrm{Mpc}$ (Fig. 5b), the points are consistent with zero at the $1 \sigma$ level despite the large scale features seen in the cone plot in Fig. 2. Other analyses not relying on the second moment of the distribution will be required to define the filamentary structures. In Fig. 5 b we compare our results with those from two other large redshift surveys. The $\xi$ calculated from the IRAS-QDOT survey (Saunders et al. 1992) shows a tendency to be higher than our own on scales of 8-30 $h^{-1} \mathrm{Mpc}$ as does that from the APM-Stromlo redshift survey (Loveday et al. 1992) although the discrepancies between the surveys are only at the $\sim 1 \sigma$ level.

The solid curve in Fig. $5 b$ is an estimate of $\xi$ derived from the angular two-point correlation function $(\omega(\theta))$ of the APM survey (Maddox et al. 1990) and the dashed line that predicted from a standard biased CDM model with $\Omega=1$ calculated using the formulation of Hamilton et al. (1991). The APM $\omega(\theta)$ apparently detects clustering above that expected by standard CDM theory to a high level of confidence although there is always the possibility of small systematic errors confusing the correlation function on larger scales. The redshift two-point correlation function however is less sensitive to such effects and the results of the completed section of the Durham/UKST survey demonstrate the potential of the whole survey as a tool for testing theoretical models such as CDM in addition to providing a clearer visual picture of large scale structure in the Universe.

\section{References}

Collins, C.A., Heydon-Dumbleton, N.H. and MacGillivray, H.T., 1988. Mon. Not. R. astron. Soc., 236, 7p.

Fairall, A.P. and Jones, A., 1988. Publs. Dept. Astr. Cape Town, 10.

Hamilton, A.J.S., Kumar, P., Lu, E. and Matthews, A., 1991. Astrophys. J., 374, L1.

Loveday, J., Efstathiou, G., Peterson, B.A. and Maddox, S.J., 1992. Astrophys. J., 400, L43.

Maddox, S.J., Efstathiou, G.P. and Sutherland, W.J., 1990. Mon. Not. R. astron. Soc., 246, 433.

Metcalfe, N., Fong, R., Shanks, T. and Kilkenny, D., 1989. Mon. Not. R. astron. Soc., 236, 207.

Parker, Q.A., 1992. In 'Digitised Optical Sky Surveys', eds. H.T. MacGillivray and E.B. Thomson, Kluwer Academic Publishers, Netherlands, p. 383.

Peterson, B.A., Ellis, R.S., Efstathiou, G.P., Shanks, T., Bean, A.J., Fong, R. and Zen-Long, Z., 1986. Mon. Not. R. astron. Soc., 221, 233.

Saunders, W., Rowan-Robinson, M and Lawrence, A., 1992. Mon. Not. R. astron. Soc., 258, 134.

Shanks, T., Bean, A.J., Efstathiou, G.P. Ellis, R.S., Fong, R. and Peterson, B.A., 1983. Astrophys. J., 274, 529.

Watson, F.G., Oates, A.P., Shanks, T. and Hale-Sutton, D., 1991. Mon. Not. R. astron. Soc., 253, 222. 